\title{
Extensions of some generalized conditions for starlikeness and convexity
}

Pranay Goswami ${ }^{1 *}$, Teodor Bulboacă² and Badr S Alkahtani ${ }^{3}$

${ }^{*}$ Correspondence:
pranaygoswami83@gmail.com
${ }^{1}$ Department of Mathematics,
School of Liberal Studies, Ambedkar
University Delhi, Delhi, 110007, India
Full list of author information is
available at the end of the article

available at the end of the article

\begin{abstract}
In the present paper we obtain generalized sufficient conditions for the starlikeness and convexity of some normalized analytic functions; the results extend those recently obtained in the work of Uyanik and Owa (J. Inequal. Appl. 2011:87, 2011).

MSC: $30 C 45$

Keywords: analytic functions; starlike and convex functions; $n$-starlike and $n$-convex functions; coefficient estimates
\end{abstract}

\section{Introduction and preliminaries}

Let $\mathcal{H}(\mathrm{U})$ be the class of holomorphic functions in the unit disk $\mathrm{U}=\{z \in \mathbb{C}:|z|<1\}$, and define

$$
\mathcal{A}_{n}=\left\{f \in \mathcal{H}(\mathrm{U}): f(z)=z+a_{n+1} z^{n+1}+\cdots, z \in \mathrm{U}\right\}, \quad n \in \mathbb{N} .
$$

Also, let

$$
\mathcal{S}_{n}^{*}=\left\{f \in \mathcal{A}_{n}: \operatorname{Re} \frac{z f^{\prime}(z)}{f(z)}>0, z \in \mathrm{U}\right\}
$$

be the class of $n$-starlike functions in $\mathrm{U}$, and

$$
\mathcal{C}_{n}=\left\{f \in \mathcal{A}_{n}: \operatorname{Re}\left(1+\frac{z f^{\prime \prime}(z)}{f^{\prime}(z)}\right)>0, z \in \mathrm{U}\right\}
$$

be the class of $n$-convex functions in $\mathrm{U}$. The notations $\mathcal{S}^{*}:=\mathcal{S}_{1}^{*}$ and $\mathcal{C}:=\mathcal{C}_{1}$ are well known, where $\mathcal{S}^{*}$ and $\mathcal{C}$ represent, respectively, the class of normalized starlike and normalized convex functions in the unit disc $\mathrm{U}$.

In the recent years, many authors have obtained some sufficient conditions for starlikeness and convexity, that can be found, for example, in [1-5] etc., together with many references. In the present paper, by using some simple integral inequalities, we generalize the results of [4] and we give some interesting special cases of our results. The methods used here are frequently used in complex analysis and operator theory on spaces of analytic functions; among others, for example, in obtaining estimations for point evaluation operators on spaces of analytic functions of one or several variables containing derivatives in the definition of the spaces (see, for example, [6] and [7]).

O2014 Goswami et al.; licensee Springer. This is an Open Access article distributed under the terms of the Creative Commons Attribution License (http://creativecommons.org/licenses/by/2.0), which permits unrestricted use, distribution, and reproduction in any medium, provided the original work is properly cited. 
To obtain the generalized conditions for $n$-starlikeness and $n$-convexity, presented in the following sections, we shall require the following lemma.

Lemma 1.1 $[1,2]$ If $f \in \mathcal{A}_{n}$ and

$$
\left|f^{\prime}(z)-1\right|<\frac{n+1}{\sqrt{(n+1)^{2}+1}}, \quad z \in \mathrm{U}
$$

then $f \in \mathcal{S}_{n}^{*}$.

\section{Generalized conditions for $\boldsymbol{n}$-starlikeness}

Using the previous lemma, we will prove the next sufficient condition for $n$-starlikeness.

Theorem 2.1 Let $f \in \mathcal{A}_{n}$ and suppose that

$$
\left|f^{\left(j_{0}\right)}(z)\right| \leq \frac{n+1}{\sqrt{(n+1)^{2}+1}}-M_{n}\left(j_{0}\right), \quad z \in \mathrm{U}
$$

for some integer $j_{0} \geq 2$, with

$$
M_{n}\left(j_{0}\right):= \begin{cases}0, & \text { if } 2 \leq j_{0} \leq n+1, \\ \sum_{k=1}^{j_{0}-n-1}\left|f^{(n+k)}(0)\right|, & \text { if } j_{0} \geq n+2 .\end{cases}
$$

Then $f \in \mathcal{S}_{n}^{*}$.

Proof Since $f \in \mathcal{A}_{n}$, we have

$$
f^{(k)}(0)=0 \quad \text { for } 2 \leq k \leq n .
$$

(i) First, we will prove that (2.1) implies $f \in \mathcal{S}_{n}^{*}$ for $j_{0}=2$. Thus, if

$$
\left|f^{\prime \prime}(z)\right| \leq \frac{n+1}{\sqrt{(n+1)^{2}+1}}, \quad z \in \mathrm{U}
$$

then we have

$$
\begin{aligned}
\left|f^{\prime}(z)-1\right| & =\left|\int_{0}^{z} f^{\prime \prime}(\zeta) d \zeta\right| \leq \int_{0}^{|z|}\left|f^{\prime \prime}\left(\rho e^{i \theta}\right)\right| d \rho \\
& \leq \frac{n+1}{\sqrt{(n+1)^{2}+1}}|z|<\frac{n+1}{\sqrt{(n+1)^{2}+1}}, \quad z \in \mathrm{U}
\end{aligned}
$$

and according to Lemma 1.1 we deduce that $f \in \mathcal{S}_{n}^{*}$.

(ii) Suppose that there exists an integer $3 \leq j_{0} \leq n+1$ such that inequality (2.1) holds. Using relation (2.3), we may easily see that

$$
\begin{aligned}
\left|f^{\left(j_{0}-1\right)}(z)\right| & =\left|\int_{0}^{z} f^{\left(j_{0}\right)}(\zeta) d \zeta+f^{\left(j_{0}-1\right)}(0)\right|=\left|\int_{0}^{z} f^{\left(j_{0}\right)}(\zeta) d \zeta\right| \\
& \leq \int_{0}^{|z|}\left|f^{\left(j_{0}\right)}\left(\rho e^{i \theta}\right)\right| d \rho \leq \frac{n+1}{\sqrt{(n+1)^{2}+1}}|z|<\frac{n+1}{\sqrt{(n+1)^{2}+1}}, \quad z \in \mathrm{U} .
\end{aligned}
$$


Consequently, if inequality (2.1) holds for some integer $3 \leq j_{0} \leq n+1$, then

$$
\left|f^{(j)}(z)\right|<\frac{n+1}{\sqrt{(n+1)^{2}+1}}, \quad z \in \mathrm{U}, \text { for all } 2 \leq j \leq j_{0}-1 .
$$

Using the fact that we have already proved that if (2.1) holds for $j_{0}=2$, then $f \in \mathcal{S}_{n}^{*}$, from the above remark it follows that our result holds for any integer $2 \leq j_{0} \leq n+1$.

(iii) Now we will prove that the result holds for $j_{0}=n+2$. Thus, a simple computation shows that

$$
\begin{aligned}
\left|f^{(n+1)}(z)\right| & =\left|\int_{0}^{z} f^{(n+2)}(\zeta) d \zeta+f^{(n+1)}(0)\right| \\
& \leq\left|\int_{0}^{z} f^{(n+2)}(\zeta) d \zeta\right|+\left|f^{(n+1)}(0)\right| \leq \int_{0}^{|z|}\left|f^{(n+2)}\left(\rho e^{i \theta}\right)\right| d \rho+\left|f^{(n+1)}(0)\right| \\
& \leq\left(\frac{n+1}{\sqrt{(n+1)^{2}+1}}-M_{n}(n+2)\right)|z|+\left|f^{(n+1)}(0)\right|<\frac{n+1}{\sqrt{(n+1)^{2}+1}}, \quad z \in \mathrm{U},
\end{aligned}
$$

that is,

$$
\left|f^{(n+1)}(z)\right|<\frac{n+1}{\sqrt{(n+1)^{2}+1}}, \quad z \in \mathrm{U}
$$

and using the result of the part (ii) of our proof for $j_{0}=n+1$, it follows that $f \in \mathcal{S}_{n}^{*}$.

(iv) Finally, supposing that there exists an integer $j_{0} \geq n+3$ such that (2.1) holds, it follows that

$$
\begin{aligned}
\left|f^{\left(j_{0}-1\right)}(z)\right| & =\left|\int_{0}^{z} f^{\left(j_{0}\right)}(\zeta) d \zeta+f^{\left(j_{0}-1\right)}(0)\right| \\
& \leq\left|\int_{0}^{z} f^{\left(j_{0}\right)}(\zeta) d \zeta\right|+\left|f^{\left(j_{0}-1\right)}(0)\right| \leq \int_{0}^{|z|}\left|f^{\left(j_{0}\right)}\left(\rho e^{i \theta}\right)\right| d \rho+\left|f^{\left(j_{0}-1\right)}(0)\right| \\
& \leq\left(\frac{n+1}{\sqrt{(n+1)^{2}+1}}-M_{n}\left(j_{0}\right)\right)|z|+\left|f^{\left(j_{0}-1\right)}(0)\right| \\
& <\frac{n+1}{\sqrt{(n+1)^{2}+1}}-M_{n}\left(j_{0}-1\right), \quad z \in \mathrm{U} .
\end{aligned}
$$

Consequently, if inequality (2.1) holds for some integer $j_{0} \geq n+3$, then

$$
\left|f^{(j)}(z)\right|<\frac{n+1}{\sqrt{(n+1)^{2}+1}}-M_{n}(j), \quad z \in \mathrm{U}, \text { for all } n+2 \leq j \leq j_{0}-1
$$

Since we have already proved that if (2.1) holds for $j_{0}=n+2$ then $f \in \mathcal{S}_{n}^{*}$, from the above remark the second part of our result follows, and the theorem is completely proved.

Remark 2.1 If we put $n=1$ in the above result, we get [4, Theorem 2.1].

\section{Generalized conditions for $\boldsymbol{n}$-convexity}

In order to prove our main result, first we give the following two lemmas. 
Lemma 3.1 Let $f \in \mathcal{A}_{n}$ and suppose that

$$
\left|f^{(j 0)}(z)\right| \leq \frac{n+1}{2 \sqrt{(n+1)^{2}+1}}, \quad z \in \mathrm{U}
$$

for some integer $2 \leq j_{0} \leq n+1$. Then $f \in \mathcal{C}_{n}$.

Proof (i) First, we will show that this implication holds for $j_{0}=2$. Letting $F(z)=z f^{\prime}(z)$, then $f \in \mathcal{C}_{n}$ if and only if $F \in \mathcal{S}_{n}^{*}$. If

$$
\left|f^{\prime \prime}(z)\right| \leq \frac{n+1}{2 \sqrt{(n+1)^{2}+1}}, \quad z \in \mathrm{U}
$$

a simple computation shows that

$$
\begin{aligned}
\left|F^{\prime}(z)-1\right| & =\left|z f^{\prime \prime}(z)+f^{\prime}(z)-1\right| \leq\left|z f^{\prime \prime}(z)\right|+\left|f^{\prime}(z)-1\right| \\
& \leq\left|z f^{\prime \prime}(z)\right|+\left|\int_{0}^{z} f^{\prime \prime}(\zeta) d \zeta\right| \leq\left|z f^{\prime \prime}(z)\right|+\int_{0}^{|z|}\left|f^{\prime \prime}\left(\rho e^{i \theta}\right)\right| d \rho \\
& \leq \frac{n+1}{2 \sqrt{(n+1)^{2}+1}}|z|+\frac{n+1}{2 \sqrt{(n+1)^{2}+1}}|z|<\frac{n+1}{\sqrt{(n+1)^{2}+1}}, \quad z \in \mathrm{U},
\end{aligned}
$$

and by Lemma 1.1 we deduce that $F \in \mathcal{S}_{n}^{*}$, i.e., $f \in \mathcal{C}_{n}$.

(ii) Suppose that there exists an integer $3 \leq j_{0} \leq n+1$ such that inequality (3.1) holds. Using relation (2.3), we may easily see that

$$
\begin{aligned}
\left|f^{\left(j_{0}-1\right)}(z)\right| & =\left|\int_{0}^{z} f^{\left(j_{0}\right)}(\zeta) d \zeta+f^{\left(j_{0}-1\right)}(0)\right|=\left|\int_{0}^{z} f^{\left(j_{0}\right)}(\zeta) d \zeta\right| \\
& \leq \int_{0}^{|z|}\left|f^{\left(j_{0}\right)}\left(\rho e^{i \theta}\right)\right| d \rho \leq \frac{n+1}{2 \sqrt{(n+1)^{2}+1}}|z|<\frac{n+1}{2 \sqrt{(n+1)^{2}+1}}, \quad z \in \mathrm{U} .
\end{aligned}
$$

Thus, we have obtained that if inequality (3.1) holds for some integer $3 \leq j_{0} \leq n+1$, then

$$
\left|f^{(j)}(z)\right|<\frac{n+1}{2 \sqrt{(n+1)^{2}+1}}, \quad z \in \mathrm{U}, \text { for all } 2 \leq j \leq j_{0}-1 .
$$

Since we have already proved that if (3.1) holds for $j_{0}=2$ then $f \in \mathcal{C}_{n}$, our result follows from the above remark.

Lemma 3.2 Let $f \in \mathcal{A}_{n}$ and suppose that

$$
\left|f^{\left(j_{0}+1\right)}(z)\right| \leq \frac{1}{j_{0}+1}\left(\frac{n+1}{\sqrt{(n+1)^{2}+1}}-N_{n}\left(j_{0}\right)\right), \quad z \in \mathrm{U},
$$

for some integer $j_{0} \geq n+1$, with

$$
N_{n}\left(j_{0}\right):=M_{n}\left(j_{0}\right)+j_{0}\left|f^{\left(j_{0}\right)}(0)\right|= \begin{cases}(n+1)\left|f^{(n+1)}(0)\right|, & \text { if } j_{0}=n+1, \\ \sum_{k=1}^{j_{0}-n-1}\left|f^{(n+k)}(0)\right|+j_{0}\left|f^{\left(j_{0}\right)}(0)\right|, & \text { if } j_{0} \geq n+2,\end{cases}
$$

where $M_{n}\left(j_{0}\right)$ is given by (2.2). Then $f \in \mathcal{C}_{n}$. 
Proof Suppose that there exists an integer $j_{0} \geq n+1$ such that inequality (3.2) holds. If we let $F(z)=z f^{\prime}(z)$, then $f \in \mathcal{C}_{n}$ if and only if $F \in \mathcal{S}_{n}^{*}$. Since

$$
F^{\left(j_{0}\right)}(z)=z f^{\left(j_{0}+1\right)}(z)+j_{0} f^{\left(j_{0}\right)}(z)
$$

we obtain that

$$
\begin{aligned}
\left|F^{\left(j_{0}\right)}(z)\right| & =\left|z f^{\left(j_{0}+1\right)}(z)+k_{0} f^{\left(j_{0}\right)}(z)\right| \\
& \leq|z| f^{\left(j_{0}+1\right)}(z)\left|+j_{0}\right| \int_{0}^{z} f^{\left(j_{0}+1\right)}(\zeta) d \zeta+f^{\left(j_{0}\right)}(0) \mid \\
& \leq|z| f^{\left(j_{0}+1\right)}(z)\left|+j_{0} \int_{0}^{|z|}\right| f^{\left(j_{0}+1\right)}\left(\rho e^{i \theta}\right)\left|d \rho+j_{0}\right| f^{\left(j_{0}\right)}(0) \mid \\
& \leq\left(j_{0}+1\right)|z| f^{\left(j_{0}+1\right)}(z)\left|+j_{0}\right| f^{\left(j_{0}\right)}(0) \mid \\
& <\frac{n+1}{\sqrt{(n+1)^{2}+1}}-N_{n}\left(j_{0}\right)+j_{0}\left|f^{\left(j_{0}\right)}(0)\right|=\frac{n+1}{\sqrt{(n+1)^{2}+1}}-M_{n}\left(j_{0}\right), \quad z \in \mathrm{U} .
\end{aligned}
$$

Thus, we have obtained that if inequality (3.2) holds for some integer $j_{0} \geq n+1$, then

$$
\left|F^{\left(j_{0}\right)}(z)\right|<\frac{n+1}{\sqrt{(n+1)^{2}+1}}-M_{n}\left(j_{0}\right), \quad z \in \mathrm{U}
$$

and from Theorem 2.1 we deduce that $F \in \mathcal{S}_{n}^{*}$, hence $f \in \mathcal{C}_{n}$, which completes the proof of the lemma.

Combining Lemma 3.1 and Lemma 3.2, we obtain the next conclusion for the $n$ convexity sufficient conditions.

Theorem 3.1 Let $f \in \mathcal{A}_{n}$ and suppose that

$$
\left|f^{\left(j_{0}+1\right)}(z)\right| \leq P_{n}\left(j_{0}\right), \quad z \in \mathrm{U}
$$

for some integer $j_{0} \geq 1$, with

$$
P_{n}\left(j_{0}\right):= \begin{cases}\frac{n+1}{2 \sqrt{(n+1)^{2}+1}}, & \text { if } 1 \leq j_{0} \leq n, \\ \frac{1}{j_{0}+1}\left(\frac{n+1}{\sqrt{(n+1)^{2}+1}}-N_{n}\left(j_{0}\right)\right), & \text { if } j_{0} \geq n+1\end{cases}
$$

where $N_{n}\left(j_{0}\right)$ is given by (3.3). Then $f \in \mathcal{C}_{n}$.

Remark 3.1 If we put $n=1$ in the above results, we obtain [4, Theorem 3.1].

Using a similar method as in the proof of Theorem 2.1 and Lemma 3.1, we can easily derive the following result.

Theorem 3.2 Let $f \in \mathcal{A}_{n}$ and suppose that

$$
\left|f^{\left(j_{0}+1\right)}(z)\right| \leq Q_{n}\left(j_{0}\right):=\frac{n+1}{2 \sqrt{(n+1)^{2}+1}}-M_{n}\left(j_{0}+1\right), \quad z \in \mathrm{U},
$$


for some integer $j_{0} \geq 1$, where $M_{n}\left(j_{0}+1\right)$ is defined by (2.2), i.e.,

$$
M_{n}\left(j_{0}+1\right)= \begin{cases}0, & \text { if } 1 \leq j_{0} \leq n \\ \sum_{k=1}^{j_{0}-n}\left|f^{(n+k)}(0)\right|, & \text { if } j_{0} \geq n+1 .\end{cases}
$$

Then $f \in \mathcal{C}_{n}$.

Proof (i) For $1 \leq j_{0} \leq n$, the result follows from Lemma 3.1.

(ii) We will prove that the result holds for $j_{0}=n+1$. A simple calculus shows that

$$
\begin{aligned}
\left|f^{(n+1)}(z)\right| & =\left|\int_{0}^{z} f^{(n+2)}(\zeta) d \zeta+f^{(n+1)}(0)\right| \\
& \leq\left|\int_{0}^{z} f^{(n+2)}(\zeta) d \zeta\right|+\left|f^{(n+1)}(0)\right| \leq \int_{0}^{|z|}\left|f^{(n+2)}\left(\rho e^{i \theta}\right)\right| d \rho+\left|f^{(n+1)}(0)\right| \\
& \leq\left(\frac{n+1}{2 \sqrt{(n+1)^{2}+1}}-M_{n}(n+2)\right)|z|+\left|f^{(n+1)}(0)\right| \\
& <\frac{n+1}{2 \sqrt{(n+1)^{2}+1}}, \quad z \in \mathrm{U},
\end{aligned}
$$

that is,

$$
\left|f^{(n+1)}(z)\right|<\frac{n+1}{2 \sqrt{(n+1)^{2}+1}}, \quad z \in \mathrm{U}
$$

and using the result of the part (i) of our proof for $j_{0}=n$, it follows that $f \in \mathcal{C}_{n}$.

(iii) Supposing that there exists an integer $j_{0} \geq n+2$ such that (3.4) holds, it follows that

$$
\begin{aligned}
\left|f^{\left(j_{0}\right)}(z)\right| & =\left|\int_{0}^{z} f^{\left(j_{0}+1\right)}(\zeta) d \zeta+f^{\left(j_{0}\right)}(0)\right| \\
& \leq\left|\int_{0}^{z} f^{\left(j_{0}+1\right)}(\zeta) d \zeta\right|+\left|f^{\left(j_{0}\right)}(0)\right| \leq \int_{0}^{|z|}\left|f^{\left(j_{0}+1\right)}\left(\rho e^{i \theta}\right)\right| d \rho+\left|f^{\left(j_{0}\right)}(0)\right| \\
& \leq\left(\frac{n+1}{2 \sqrt{(n+1)^{2}+1}}-M_{n}\left(j_{0}+1\right)\right)|z|+\left|f^{\left(j_{0}\right)}(0)\right| \\
& <\frac{n+1}{2 \sqrt{(n+1)^{2}+1}}-M_{n}\left(j_{0}\right), \quad z \in \mathrm{U} .
\end{aligned}
$$

Thus, if inequality (3.4) holds for some integer $j_{0} \geq n+2$, then

$$
\left|f^{(j+1)}(z)\right|<\frac{n+1}{2 \sqrt{(n+1)^{2}+1}}-M_{n}(j+1), \quad z \in \mathrm{U}, \text { for all } n+1 \leq j \leq j_{0} .
$$

Since we have already proved to the part (ii) that if (3.4) holds for $j_{0}=n+1$ then $f \in \mathcal{C}_{n}$, our theorem follows from the above remark.

Remark 3.2 If we put $n=1$ in the above results, then we get [4, Theorem 3.2]. 
It remains to determine which of the above last two theorems gives weaker sufficient conditions for $n$-convexity, i.e.,

$$
\left|f^{\left(j_{0}+1\right)}(z)\right| \leq R_{n}\left(j_{0}\right):=\max \left\{P_{n}\left(j_{0}\right) ; Q_{n}\left(j_{0}\right)\right\}, \quad z \in \mathrm{U}
$$

implies $f \in \mathcal{C}_{n}$.

(i) First, let us consider the case $j_{0} \geq n+2$.

A simple computation shows that $R_{n}\left(j_{0}\right)=Q_{n}\left(j_{0}\right)$ if and only if

$$
Q_{n}\left(j_{0}\right) \geq P_{n}\left(j_{0}\right) \quad \text { and } \quad Q_{n}\left(j_{0}\right) \geq 0
$$

which is equivalent to $M_{n}\left(j_{0}+1\right) \leq \min \{A ; B\}$, where

$$
A:=\frac{j_{0}-1}{j_{0}}\left(\frac{n+1}{2 \sqrt{(n+1)^{2}+1}}+\left|f^{\left(j_{0}\right)}(0)\right|\right), \quad B:=\frac{n+1}{2 \sqrt{(n+1)^{2}+1}} .
$$

Moreover, it is easy to check that

$$
\min \{A ; B\}=A \quad \Leftrightarrow \quad\left(j_{0}-1\right)\left|f^{\left(j_{0}\right)}(0)\right| \leq \frac{n+1}{2 \sqrt{(n+1)^{2}+1}},
$$

and

$$
\min \{A ; B\}=B \quad \Leftrightarrow \quad\left(j_{0}-1\right)\left|f^{\left(j_{0}\right)}(0)\right| \geq \frac{n+1}{2 \sqrt{(n+1)^{2}+1}} .
$$

Reversely, $R_{n}\left(j_{0}\right)=P_{n}\left(j_{0}\right)$ if and only if

$$
Q_{n}\left(j_{0}\right) \leq P_{n}\left(j_{0}\right) \quad \text { and } \quad P_{n}\left(j_{0}\right) \geq 0 \text {, }
$$

that holds whenever $A \leq M_{n}\left(j_{0}+1\right) \leq C$, where

$$
C:=\frac{n+1}{\sqrt{(n+1)^{2}+1}}-\left(j_{0}-1\right)\left|f^{\left(j_{0}\right)}(0)\right|
$$

Moreover, we have

$$
A \leq C \quad \Leftrightarrow \quad\left(j_{0}-1\right)\left|f^{\left(j_{0}\right)}(0)\right| \geq \frac{n+1}{2 \sqrt{(n+1)^{2}+1}} .
$$

(ii) Secondly, let us consider the case $j_{0}=n+1$.

A simple calculus shows that

$$
R_{n}(n+1)=Q_{n}(n+1)=\frac{n+1}{2 \sqrt{(n+1)^{2}+1}}-\left|f^{(n+1)}(0)\right|
$$

if and only if

$$
Q_{n}(n+1) \geq P_{n}(n+1) \quad \text { and } \quad Q_{n}(n+1) \geq 0 \text {, }
$$


which is equivalent to

$$
\left|f^{(n+1)}(0)\right| \leq \min \left\{\frac{n(n+1)}{2 \sqrt{(n+1)^{2}+1}} ; \frac{n+1}{2 \sqrt{(n+1)^{2}+1}}\right\}=\frac{n+1}{2 \sqrt{(n+1)^{2}+1}} .
$$

Reversely,

$$
R_{n}(n+1)=P_{n}(n+1)=\frac{n+1}{n+2}\left(\frac{n+1}{\sqrt{(n+1)^{2}+1}}-\left|f^{(n+1)}(0)\right|\right)
$$

if and only if

$$
Q_{n}(n+1) \leq P_{n}(n+1) \quad \text { and } \quad P_{n}(n+1) \geq 0,
$$

that holds whenever

$$
\frac{n(n+1)}{2 \sqrt{(n+1)^{2}+1}} \leq\left|f^{(n+1)}(0)\right| \leq \frac{1}{\sqrt{(n+1)^{2}+1}} .
$$

In order to have

$$
\frac{n(n+1)}{2 \sqrt{(n+1)^{2}+1}} \leq \frac{1}{\sqrt{(n+1)^{2}+1}}
$$

we obtain that $n=1$, but this case is contained in the first part of (ii).

Now, from Theorem 3.1 and Theorem 3.2, we deduce the next conclusion.

Theorem 3.3 Let $f \in \mathcal{A}_{n}$ and suppose that

$$
\left|f^{\left(j_{0}+1\right)}(z)\right| \leq R_{n}\left(j_{0}\right), \quad z \in \mathrm{U},
$$

for some integer $j_{0} \geq 1$, with

(i) $\quad R_{n}\left(j_{0}\right):=\frac{n+1}{2 \sqrt{(n+1)^{2}+1}}, \quad$ if $1 \leq j_{0} \leq n$,

(ii) $\quad R_{n}\left(j_{0}\right):=\frac{n+1}{2 \sqrt{(n+1)^{2}+1}}-\left|f^{(n+1)}(0)\right|, \quad$ if $j_{0}=n+1$

$$
\text { and }\left|f^{(n+1)}(0)\right| \leq \frac{n+1}{2 \sqrt{(n+1)^{2}+1}} \text {, }
$$

(iii) $\quad R_{n}\left(j_{0}\right):=\frac{n+1}{2 \sqrt{(n+1)^{2}+1}}-M_{n}\left(j_{0}+1\right), \quad$ if $j_{0} \geq n+2$,

$$
\left(j_{0}-1\right)\left|f^{\left(j_{0}+1\right)}(0)\right|>\frac{n+1}{2 \sqrt{(n+1)^{2}+1}} \text { and } M_{n}\left(j_{0}+1\right) \leq B,
$$

(iv) $R_{n}\left(j_{0}\right):=\frac{n+1}{2 \sqrt{(n+1)^{2}+1}}-M_{n}\left(j_{0}+1\right), \quad$ if $j_{0} \geq n+2$,

$$
\left(j_{0}-1\right)\left|f^{\left(j_{0}+1\right)}(0)\right| \leq \frac{n+1}{2 \sqrt{(n+1)^{2}+1}} \text { and } M_{n}\left(j_{0}+1\right) \leq A \text {, }
$$


(v) $R_{n}\left(j_{0}\right):=\frac{1}{j_{0}+1}\left(\frac{n+1}{\sqrt{(n+1)^{2}+1}}-M_{n}\left(j_{0}+1\right)-\left(j_{0}-1\right)\left|f^{\left(j_{0}\right)}(0)\right|\right)$,

$$
\text { if } j_{0} \geq n+2,\left(j_{0}-1\right)\left|f^{\left(j_{0}+1\right)}(0)\right| \leq \frac{n+1}{2 \sqrt{(n+1)^{2}+1}} \text { and } A \leq M_{n}\left(j_{0}+1\right) \leq C \text {, }
$$

where $M_{n}\left(j_{0}\right), A, B$ and $C$ are given by (2.2), (3.5) and (3.6), respectively. Then $f \in \mathcal{C}_{n}$.

\section{Special cases}

In this section we give some special cases of our main results.

1. Taking the polynomial function

$$
f_{n, m}(z)=z+a_{n+1} z^{n+1}+\cdots+a_{n+m} z^{n+m}, \quad z \in \mathbb{C},
$$

where $m \in \mathbb{N}$, then $f_{n, m} \in \mathcal{A}_{n}, \operatorname{deg} f_{n, m}=n+m$. It is easy to see that

$$
\begin{aligned}
& f_{n, m}^{\left(j_{0}\right)}(z)=j_{0} ! \sum_{k=1}^{m}\left(\begin{array}{c}
n+k \\
j_{0}
\end{array}\right) a_{n+k} z^{n-j_{0}+k}, \quad z \in \mathbb{C}, \text { if } 2 \leq j_{0} \leq n+1, \\
& f_{n, m}^{\left(j_{0}\right)}(z)=j_{0} ! \sum_{k=j_{0}-n}^{n-j_{0}+k}\left(\begin{array}{c}
n+k \\
n-j_{0}+k
\end{array}\right) a_{n+k} z^{n-j_{0}+k}, \quad z \in \mathbb{C}, \text { if } n+2 \leq j_{0} \leq n+m,
\end{aligned}
$$

and from Theorem 2.1 we deduce the following result.

Corollary 4.1 Let $f_{n, m}$ be the polynomial function of the form (4.1) and suppose that for some integer $2 \leq j_{0} \leq n+m$, one of the following inequalities holds:

(i) $s_{n}^{\left(j_{0}\right)}(m) \leq \frac{n+1}{\sqrt{(n+1)^{2}+1}}, \quad$ if $2 \leq j_{0} \leq n+1$,

(ii) $s_{n}^{\left(j_{0}\right)}(m) \leq \frac{n+1}{\sqrt{(n+1)^{2}+1}}-\sum_{j=1}^{j_{0}-n-1}(n+j) !\left|a_{n+j}\right|, \quad$ if $n+2 \leq j_{0} \leq n+m$,

where

$$
\begin{aligned}
& s_{n}^{\left(j_{0}\right)}(m)=j_{0} ! \sum_{k=1}^{m}\left(\begin{array}{c}
n+k \\
j_{0}
\end{array}\right)\left|a_{n+k}\right|, \quad \text { if } 2 \leq j_{0} \leq n+1, \\
& s_{n}^{\left(j_{0}\right)}(m)=j_{0} ! \sum_{k=j_{0}-n}^{m}\left(\begin{array}{c}
n+k \\
n-j_{0}+k
\end{array}\right)\left|a_{n+k}\right|, \quad \text { if } n+2 \leq j_{0} \leq n+m .
\end{aligned}
$$

Then $f_{n, m} \in \mathcal{S}_{n}^{*}$.

Taking $j_{0}=n+1$ and $j_{0}=n+m$ in Corollary 4.1, we deduce that if one of the following inequalities holds:

$$
\begin{aligned}
& (n+1) ! \sum_{k=1}^{m}\left(\begin{array}{c}
n+k \\
n+1
\end{array}\right)\left|a_{n+k}\right| \leq \frac{n+1}{\sqrt{(n+1)^{2}+1}} \\
& \sum_{k=1}^{m}(n+k) !\left|a_{n+k}\right| \leq \frac{n+1}{\sqrt{(n+1)^{2}+1}}
\end{aligned}
$$


then $f_{n, m} \in \mathcal{S}_{n}^{*}$. It is easy to check that condition (4.6) is stronger than (4.7), hence we get the following.

Example 4.1 If $f_{n, m}$ is the polynomial function of the form (4.1) and

$$
\sum_{k=1}^{m}(n+k) !\left|a_{n+k}\right| \leq \frac{n+1}{\sqrt{(n+1)^{2}+1}}
$$

then $f_{n, m} \in \mathcal{S}_{n}^{*}$.

Remark 4.1 For $n=1$ and $m=3$, the above example reduces to the [4, Example 2.1], that is, if $\left|a_{2}\right|+3\left|a_{3}\right|+12\left|a_{4}\right| \leq \frac{1}{\sqrt{5}}$, then $f_{1,3}(z)=z+a_{2} z^{2}+a_{3} z^{3}+a_{4} z^{4} \in \mathcal{S}_{n}^{*}$.

According to Theorem 3.3 and using the differentiation formulas (4.2) and (4.3), we obtain the next result.

Corollary 4.2 Let $f_{n, m}$ be the polynomial function of the form (4.1) and suppose that for some integer $1 \leq j_{0} \leq n+m-1$, one of the following inequalities holds:

(i) $\quad s_{n}^{\left(j_{0}+1\right)}(m) \leq \frac{n+1}{2 \sqrt{(n+1)^{2}+1}}, \quad$ if $1 \leq j_{0} \leq n$,

(ii) $\quad s_{n}^{\left(j_{0}+1\right)}(m) \leq \frac{n+1}{2 \sqrt{(n+1)^{2}+1}}-(n+1) !\left|a_{n+1}\right|, \quad$ if $j_{0}=n+1$,

(iii) $\quad s_{n}^{\left(j_{0}+1\right)}(m) \leq \frac{n+1}{2 \sqrt{(n+1)^{2}+1}}-\sum_{j=1}^{j_{0}-n}(n+j) !\left|a_{n+j}\right|$,

$$
\begin{aligned}
& \text { if } n+2 \leq j_{0} \leq n+m-1,\left(j_{0}+1\right) !\left(j_{0}-1\right)\left|a_{j_{0}+1}\right|>\frac{n+1}{2 \sqrt{(n+1)^{2}+1}} \\
& \text { and } \sum_{j=1}^{j_{0}-n}(n+j) !\left|a_{n+j}\right| \leq B^{\prime}
\end{aligned}
$$

(iv) $\quad s_{n}^{\left(j_{0}+1\right)}(m) \leq \frac{n+1}{2 \sqrt{(n+1)^{2}+1}}-\sum_{j=1}^{j_{0}-n}(n+j) !\left|a_{n+j}\right|$,

$$
\begin{aligned}
& \text { if } n+2 \leq j_{0} \leq n+m-1,\left(j_{0}+1\right) !\left(j_{0}-1\right)\left|a_{j_{0}+1}\right| \leq \frac{n+1}{2 \sqrt{(n+1)^{2}+1}}, \\
& \text { and } \sum_{j=1}^{j_{0}-n}(n+j) !\left|a_{n+j}\right| \leq A^{\prime}
\end{aligned}
$$

(v) $s_{n}^{\left(j_{0}+1\right)}(m) \leq \frac{1}{j_{0}+1}\left(\frac{n+1}{2 \sqrt{(n+1)^{2}+1}}-\sum_{j=1}^{j_{0}-n}(n+j) !\left|a_{n+j}\right|-j_{0} !\left(j_{0}-1\right)\left|a_{j_{0}}\right|\right)$,

$$
\begin{aligned}
& \text { if } n+2 \leq j_{0} \leq n+m-1,\left(j_{0}+1\right) !\left(j_{0}-1\right)\left|a_{j_{0}+1}\right| \leq \frac{n+1}{2 \sqrt{(n+1)^{2}+1}} \\
& \text { and } A^{\prime} \leq \sum_{j=1}^{j_{0}-n}(n+j) !\left|a_{n+j}\right| \leq C^{\prime}
\end{aligned}
$$


where $s_{n}^{\left(j_{0}+1\right)}(m)$ are given by (4.4) and (4.5), and

$$
\begin{aligned}
& A^{\prime}:=\frac{j_{0}-1}{j_{0}}\left(\frac{n+1}{2 \sqrt{(n+1)^{2}+1}}+j_{0} !\left|a_{j_{0}}\right|\right), \\
& B^{\prime}:=\frac{n+1}{2 \sqrt{(n+1)^{2}+1}}, \quad C^{\prime}:=\frac{n+1}{\sqrt{(n+1)^{2}+1}}-j_{0} !\left(j_{0}-1\right)\left|a_{j_{0}}\right| .
\end{aligned}
$$

Then $f_{n, m} \in \mathcal{C}_{n}$.

Taking $j_{0}=n$ in Corollary 4.2, we deduce the following special case.

Example 4.2 If $f_{n, m}$ is the polynomial function of the form (4.1) and

$$
(n+1) ! \sum_{k=1}^{m}\left(\begin{array}{l}
n+k \\
n+1
\end{array}\right)\left|a_{n+k}\right| \leq \frac{n+1}{2 \sqrt{(n+1)^{2}+1}}
$$

then $f_{n, m} \in \mathcal{C}_{n}$.

Remark 4.2 For $n=1$ and $m=3$, the above example reduces to the following one: if $\left|a_{2}\right|+$ $3\left|a_{3}\right|+6\left|a_{4}\right| \leq \frac{1}{2 \sqrt{5}}$, then $f_{1,3}(z)=z+a_{2} z^{2}+a_{3} z^{3}+a_{4} z^{4} \in \mathcal{C}_{n}$.

2. For the function

$$
f_{n}(z)=z+\alpha\left(e^{z}-\sum_{k=0}^{n} \frac{z^{k}}{k !}\right)=z+\sum_{k=1}^{\infty} \frac{\alpha}{(n+k) !} z^{n+k}, \quad z \in \mathbb{C}
$$

where $\alpha \in \mathbb{C}$, we have $f_{n} \in \mathcal{A}_{n}$, and we may easily check that

$$
\left|f_{n}^{(n+1)}(z)\right|=\left|\alpha \sum_{k=1}^{\infty} \frac{z^{k-1}}{(k-1) !}\right| \leq|\alpha| \sum_{k=1}^{\infty} \frac{1}{(k-1) !}=|\alpha| e, \quad z \in \mathrm{U}
$$

If we take $j_{0}=n+1$ in Theorem 2.1 and $j_{0}=n$ in Theorem 3.3, we deduce, respectively, the following.

Example 4.3 If $f_{n}$ is the function defined by (4.8) and

$$
|\alpha| \leq \frac{n+1}{e \sqrt{(n+1)^{2}+1}}
$$

then $f_{n} \in \mathcal{S}_{n}^{*}$.

Example 4.4 If $f_{n}$ is the function of the form (4.8) and

$$
|\alpha| \leq \frac{n+1}{2 e \sqrt{(n+1)^{2}+1}}
$$

then $f_{n} \in \mathcal{C}_{n}^{*}$. 
3. Considering the function

$$
\begin{aligned}
g_{2 n}(z) & =z+\alpha\left(\sin z-\sum_{k=0}^{n-1}(-1)^{k} \frac{z^{2 k+1}}{(2 k+1) !}\right) \\
& =z+\sum_{k=n}^{\infty} \frac{(-1)^{k} \alpha}{(2 k+1) !} z^{2 k+1}, \quad z \in \mathbb{C},
\end{aligned}
$$

where $\alpha \in \mathbb{C}$, then $g_{2 n} \in \mathcal{A}_{2 n}$, and

$$
\begin{aligned}
\left|g_{2 n}^{(2 n+1)}(z)\right| & =\left|\alpha \sum_{j=0}^{\infty}(-1)^{n+j} \frac{z^{2 j}}{(2 j) !}\right| \\
& \leq|\alpha| \sum_{j=0}^{\infty} \frac{1}{(2 j) !}=|\alpha| \frac{e+e^{-1}}{2}, \quad z \in \mathrm{U} .
\end{aligned}
$$

Taking $j_{0}=2 n+1$ in Theorem 2.1 and $j_{0}=2 n$ in Theorem 3.3, we obtain, respectively, the following.

Example 4.5 If $g_{2 n}$ is the function defined by (4.9) and

$$
|\alpha| \leq \frac{2}{e+e^{-1}} \frac{2 n+1}{\sqrt{(2 n+1)^{2}+1}}
$$

then $g_{2 n} \in \mathcal{S}_{2 n}^{*}$.

Example 4.6 If $g_{2 n}$ is the function of the form (4.9) and

$$
|\alpha| \leq \frac{1}{e+e^{-1}} \frac{2 n+1}{\sqrt{(2 n+1)^{2}+1}},
$$

then $g_{2 n} \in \mathcal{C}_{2 n}^{*}$.

4. Taking the function

$$
\begin{aligned}
h_{2 n+1}(z) & =z+\alpha\left(\cos z-\sum_{k=0}^{n}(-1)^{k} \frac{z^{2 k}}{(2 k) !}\right) \\
& =z+\sum_{j=1}^{\infty} \frac{(-1)^{n+j} \alpha}{(2 n+2 j) !} z^{2 n+2 j}, \quad z \in \mathbb{C},
\end{aligned}
$$

where $\alpha \in \mathbb{C}$, then $h_{2 n+1} \in \mathcal{A}_{2 n+1}$, and

$$
\begin{aligned}
\left|h_{2 n+1}^{(2 n+2)}(z)\right| & =\left|\alpha \sum_{j=1}^{\infty}(-1)^{n+j} \frac{z^{2 j-2}}{(2 j-2) !}\right| \\
& \leq|\alpha| \sum_{j=1}^{\infty} \frac{1}{(2 j-2) !}=|\alpha| \frac{e+e^{-1}}{2}, \quad z \in \mathrm{U} .
\end{aligned}
$$


If we choose $j_{0}=2 n+2$ in Theorem 2.1 and $j_{0}=2 n+1$ in Theorem 3.3, we obtain, respectively, the following.

Example 4.7 If $h_{2 n+1}$ is the function defined by (4.10) and

$$
|\alpha| \leq \frac{2}{e+e^{-1}} \frac{2(n+1)}{\sqrt{4(n+1)^{2}+1}},
$$

then $h_{2 n+1} \in \mathcal{S}_{2 n+1}^{*}$.

Example 4.8 If $h_{2 n+1}$ is the function of the form (4.10) and

$$
|\alpha| \leq \frac{1}{e+e^{-1}} \frac{2(n+1)}{\sqrt{4(n+1)^{2}+1}}
$$

then $h_{2 n+1} \in \mathcal{C}_{2 n+1}^{*}$.

We emphasize that all the above $n$-starlikeness and $n$-convexity criteria are very useful since the direct proofs are too difficult in each of these examples.

\section{Competing interests}

The authors declare that they have no competing interests.

\section{Authors' contributions}

All authors contributed equally to the writing of this paper. All authors read and approved the final manuscript.

\section{Author details}

${ }^{1}$ Department of Mathematics, School of Liberal Studies, Ambedkar University Delhi, Delhi, 110007, India. ${ }^{2}$ Faculty of Mathematics and Computer Science, Babeş-Bolyai University, Cluj-Napoca, 400084, Romania. ${ }^{3}$ Department of Mathematics, College of Science, King Saud University, P.O. Box 1142, Riyadh, 11989, Saudi Arabia.

\section{Acknowledgements}

Authors are thankful to reviewers for careful reading of the paper. The third author BS Alkahtani is grateful to King Saud University, Deanship of Scientific Research, College of Science Research Center to support this project.

Received: 25 September 2014 Accepted: 16 October 2014 Published: 31 Oct 2014

\section{References}

1. Mocanu, PT: Some simple criteria for starlikeness and convexity. Libertas Math. 13, 27-40 (1993)

2. Mocanu, PT, Oros, GI: A sufficient condition for starlikeness of order $\alpha$. Int. J. Math. Math. Sci. 28(9), 557-560 (2001)

3. Nunokawa, M, Owa, S, Polatoglu, Y, Çaglar, M, Duman, EY: Some sufficient conditions for starlikeness and convexity. Turk. J. Math. 34, 333-337 (2010)

4. Uyanik, N, Owa, S: Generalized conditions for starlikeness and convexity for certain analytic functions. J. Inequal. Appl. 2011, Article ID 87 (2011)

5. Goyal, SP, Bansal, SK, Goswami, P: Extensions of sufficient conditions for starlikeness and convexity of order $\alpha$ for multivalent function. Appl. Math. Lett. 9, 557-560 (2012)

6. Li, S, Stević, S: Volterra type operators on Zygmund space. J. Inequal. Appl. 2007, Article ID 32124 (2007)

7. Stević, S: On an integral operator on the unit ball in $\mathbb{C}^{n}$. J. Inequal. Appl. 1, 81-88 (2005)

10.1186/1029-242X-2014-431

Cite this article as: Goswami et al.: Extensions of some generalized conditions for starlikeness and convexity. Journal of Inequalities and Applications 2014, 2014:431 